\title{
The latency of circular vection during different accelerations of the optokinetic stimulus
}

\author{
G. A. MELCHER and V. HENN \\ Department of Neurology, University of Zürich, Zürich, Switzerland
}

\begin{abstract}
Subjects were seated inside a full-field optokinetic cylinder which was accelerated with values between .1 and $100 \mathrm{deg} / \mathrm{sec}^{2}$. Subjects indicated when motion was first detected. Latency for onset of self-motion shows a minimum of around $5 \mathrm{deg} / \mathrm{sec}^{2}$ and increases for lower and faster accelerations of the visual surround. In the low acceleration range, up to $5 \mathrm{deg} / \mathrm{sec}^{2}$, all movement is perceived as circular vection, that is, self-rotation. With higher accelerations, motion of the visual surround is perceived initially; over seconds, this gradually transforms to circular vection. Velocity estimation during low acceleration is better than during comparable vestibular acceleration. During subject rotation in the light, that is, when both the visual and vestibular inputs combine to generate a velocity signal, detection of motion has the shortest latency and represents actual velocity over a wider range than it does with each stimulus alone.
\end{abstract}

The sensation of circular vection (CV), that is, selfrotation, can be induced by full-field optokinetic stimulation (Dichgans \& Brandt, 1978; Fischer \& Kornmüller, 1930; Mach, 1875/1967). Movement of an optokinetic cylinder totally enclosing the subject is regarded as an optimal stimulus, although $\mathrm{CV}$ can also be elicited with smaller areas of visual stimulation (Brandt et al., 1973). With full-field stimulation, a rather precise correlation can be established between stimulus velocity, subjective motion sensation, and nystagmus velocity. When a subject is exposed suddenly to a moving stimulus, it usually takes a few seconds for CV to develop fully. This has been demonstrated in experiments in which the optokinetic drum was accelerated in darkness to different constant velocities before the lights were turned on (Brandt et al., 1973). This would correspond to a step in angular velocity with infinitely high acceleration.

The present study was undertaken to investigate the influence of acceleration of the visual stimulus on the occurrence, latency, and buildup of CV. These values were compared with motion sensation during vestibular stimulation in darkness or during combined visual-vestibular stimulation. This would permit an estimation of the contribution of $\mathrm{CV}$ to motion sensation during combined visual-vestibular stimulation. A comparison was also to be made with nystagmus and concomitant single-unit activity in the vestibular nuclei of monkeys exposed to identical stimulation.

\section{METHODS}

Twenty-two human subjects participated; two of them had had previous experience with $\mathrm{CV}$ in the laboratory. Their ages ranged from 16 to 68 years, with a mean of 28 years.

Correspondence should be directed to $V$. Henn, Neurological Clinic, University Hospital, CH-8091 Zürich, Switzerland.
The subjects indicated subjective sensation by rotating a handle fixed to the shaft of a potentiometer. Changes of position were indicated continuously by movement of the handle through a corresponding angle. One full subjective rotation was indicated by a full revolution of the handle. The start of self-motion was separately indicated by a buttonpress. In addition, the subjects reported their experience verbally.

The subjects were seated on a servocontrolled turntable driven by a torque motor. The turntable was totally enclosed by a rotatable, 140-cm-diam optokinetic cylinder with attached top and bottom. During optokinetic stimulation, the cylinder, which was controlled by a second torque motor, was illuminated from within and rotated around the stationary subject. The cylinder accurately followed accelerations of $.1-160 \mathrm{deg} / \mathrm{sec}^{2}$. During vestibular stimulation, the subject was rotated in the dark with the surrounding optokinetic cylinder serving as a lightproof enclosure. During combined stimulation, the subject was rotated inside the nonmoving illuminated cylinder. Amount and start of subjective motion sensation and of turntable and cylinder acceleration and position were recorded on a six-channel rectilinear oscillograph with a paper speed of $10 \mathrm{~mm} / \mathrm{sec}$. All measurements were made from these paper charts.

Latencies for the detection of motion were measured for all subjects. The subjects were asked to indicate by pressing a button the moment they started to experience self-motion. The acceleration used in different trials varied between .1 and $100 \mathrm{deg} / \mathrm{sec}^{2}$ and lasted between 1.5 (highest acceleration) and $100 \mathrm{sec}$ (lowest acceleration), reaching maximum velocities between 10 and $150 \mathrm{deg} / \mathrm{sec}$ before deceleration. The subject was accelerated in either direction in random order. The conditions-optokinetic, vestibular, and combined stimulation-were also randomized.

Six subjects were selected on the basis of their ability to indicate the amount of subjective motion sensation reliably. In these subjects, in addition to latencies, the instantaneous subjective velocity was measured at different stimulus velocities. This was done by determining the rate of change in subjective position.

\section{RESULTS}

\section{Experiment 1: Latency Measurements}

Latency to optokinetic stimulation. All subjects experienced circular vection (CV), which was always in the direction opposite to that of cylinder rotation. In the trials with low acceleration, all motion was 
interpreted as full CV, with accelerations of $10 \mathrm{deg} /$ $\sec ^{2}$ and above, more and more subjects experienced the cylinder as initially moving. It then took up to several seconds for all motion to be perceived as CV and the cylinder to be regarded as stationary. This conversion from object-motion to self-motion was always gradual.

An example is given in Figure 1. In $\mathbf{A}$, the optokinetic cylinder was accelerated by $.5 \mathrm{deg} / \mathrm{sec}^{2}$. With a latency of $2.1 \mathrm{sec}$, full CV was indicated. The velocity of $\mathrm{CV}$ increased approximately with the velocity of cylinder rotation, which can be seen clearly in B with an acceleration of $10 \mathrm{deg} / \mathrm{sec}^{2}$.

The latencies for the detection of $\mathrm{CV}$ were longest for small accelerations, and decreased to a minimum value of $1.4 \mathrm{sec}$, on average, for accelerations of $5 \mathrm{deg} / \mathrm{sec}^{2}$. In this low acceleration range, the first detection of motion was always full $\mathrm{CV}$ and not cylinder motion. At accelerations above $5 \mathrm{deg} / \mathrm{sec}^{2}$, la-
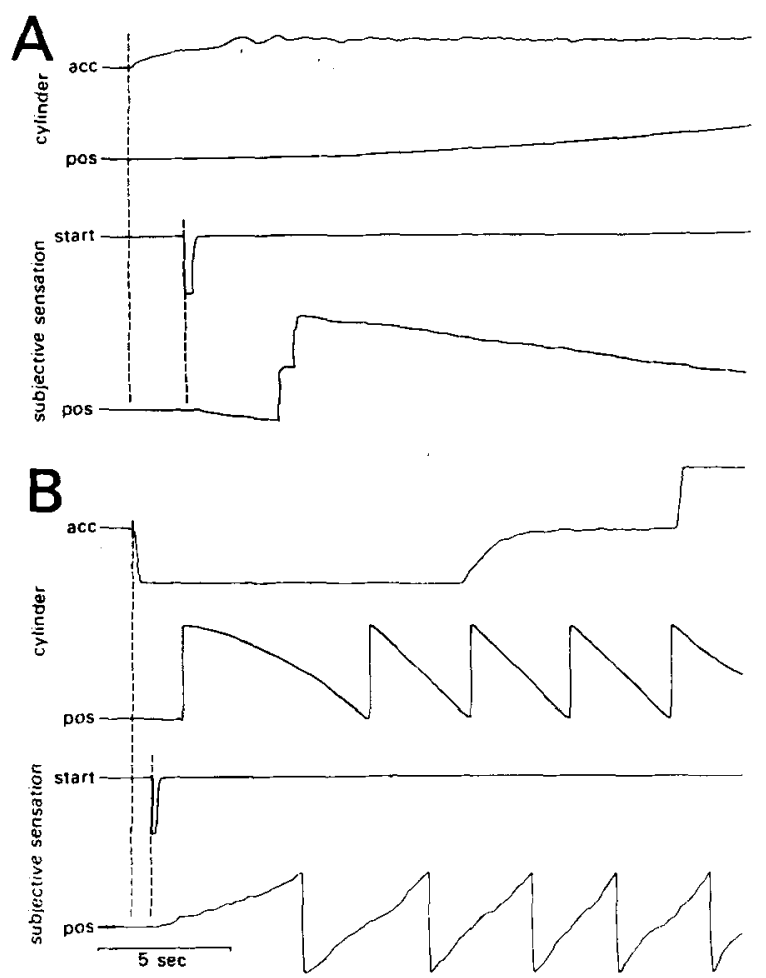

Figure 1. Latency and velocity estimation during optokinetic stimulation with a visual acceleration of $.5 \mathrm{deg} / \mathrm{sec}^{2}$ in $A$ and $10 \mathrm{deg} / \mathrm{sec}^{2}$ in B. In A, from top down, are indicated cylinder acceleration (high gain, peak clipped at an arbitrary level), cylinder position (potentiometer with reset every 360 deg mounted on the cylinder shaft), push-button indicator for first sign of $\mathrm{CV}$, and subjective position (potentiometer rotated by the subject with a reset every $360 \mathrm{deg}$ ). The subject signaled the occurrence of $\mathrm{CV}$ (third line) with a latency of $2.1 \mathrm{sec}$ in $A$ and $.8 \mathrm{sec}$ in $B$ after the start of cylinder motion. The subject also continuously indicated subjective position, which changes approximately at the same rate as cylinder position, but in the opposite direction. tencies for the first detection of $\mathrm{CV}$ increased again to around $2 \mathrm{sec}$ (at $100 \mathrm{deg} / \mathrm{sec}^{2}$ ). Then the initial cylinder rotation was often perceived as object motion which only gradually, over 1-2 sec, transformed into selfmotion. Values for one subject are shown in Figure 2A, and averages for all subjects, in Figure 2B. Single values for the latency of detection of the first sign of motion are shown in Figure 3 for all subjects. On average, the latency for the detection of $\mathrm{CV}$ has a minimum at $5 \mathrm{deg} / \mathrm{sec}^{2}$. At acceleration values below that, there is a monotonic increase in latencies for every subject. At values above, there is a tendency for a latency increase that is more variable. At these higher accelerations, some of the naive subjects occasionally reported confusion about the exact onset of object or self-motion.

Latency to vestibular stimulation. It is already known from the literature that latency for the detection of self-motion decreases with increasing acceleration values (summary: Guedry, 1974). This is true for individuals as well as for averaged values. In our sample, five subjects experienced motion at an acceleration of $.1 \mathrm{deg} / \mathrm{sec}^{2}$ with a latency of $11 \mathrm{sec}$, and all subjects at $1 \mathrm{deg} / \mathrm{sec}^{2}$ with a latency of $6.9 \mathrm{sec}$ (Figure 2B). Therefore, no standard deviations are given for values below $1 \mathrm{deg} / \mathrm{sec}^{2}$. At $5 \mathrm{deg} / \mathrm{sec}^{2}$, the latency for detection of motion is still longer for the detection of a pure vestibular stimulus- $2.0 \mathrm{sec}$, compared with $1.4 \mathrm{sec}$ for optokinetic stimulationwhereas at $10 \mathrm{deg} / \mathrm{sec}^{2}$ and above, this relationship is inverted $-1.0 \mathrm{sec}$ vestibular and $1.6 \mathrm{sec}$ optokinetic.

Latency to combined stimulation. All subjects indicated that the shortest latencies at all accelerations occurred during acceleration inside the illuminated nonmoving optokinetic cylinder (Figure 2B). In the high acceleration range above $5 \mathrm{deg} / \mathrm{sec}^{2}$, the latencies are similar to those seen in pure vestibular stimulation in the dark. In the low acceleration range, the values are close to those of pure optokinetic stimulation, but tend to be still shorter.

\section{Experiment 2: Subjective Velocity Estimation}

Six subjects, whose subjective responses were known to be reliable, were selected to continuously indicate subjective velocity under different stimulus conditions!

Optokinetic stimulation. The subjects continuously indicated the amount of CV by rotating the potentiometer handle while the optokinetic cylinder was accelerated with different values. Subjective velocities relative to stimulus velocities are shown in Figure $4 \mathrm{~A}$. It is important to realize that the abscissa is a velocity, not a time scale. Within the velocity range up to $30 \mathrm{deg} / \mathrm{sec}$, during low accelerations, subjective velocity closely corresponds to instantaneous stimulus velocity. At higher accelerations, subjective velocity initially lags stimulus velocity and remains 

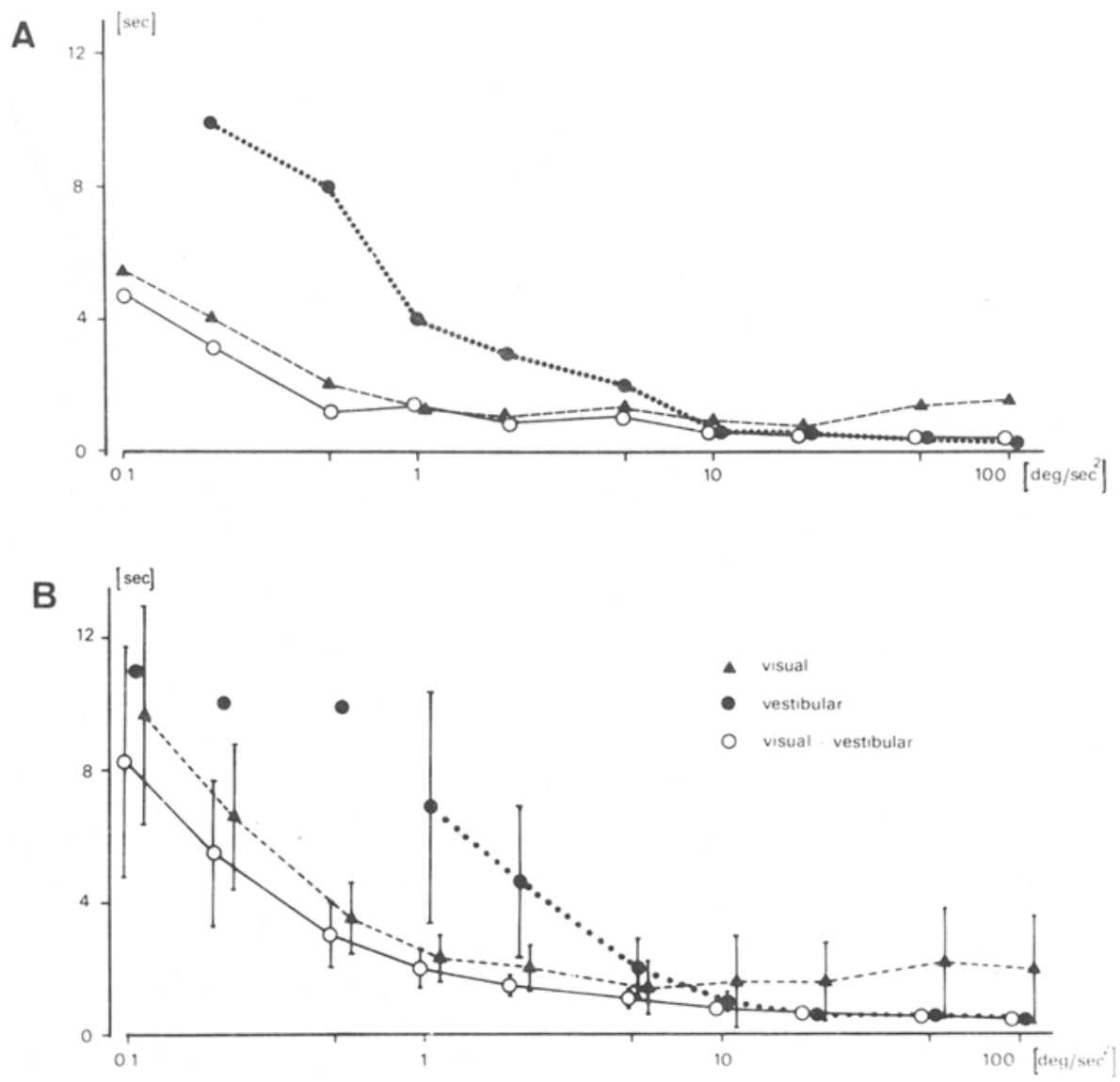

Figure 2. Latencies for the detection of motion (first sign of self-motion) during optokinetic (triangles), vestibular (dots), and combined stimulation (open circles) at different accelerations for one subject $(A)$ and averaged for all subjects $(B)$. The abscissa gives values of respective accelerations, the ordinate, the latency in seconds. Up to $5 \mathrm{deg} / \mathrm{sec}^{2}$, latencies are shorter for optokinetic than vestibular stimulation; at $10 \mathrm{deg} / \mathrm{sec}^{2}$ and above, this relation reverses. For combined stimulation, latencles have the shortest values at all accelerations. For low vestibular accelerations, averages (without standard deviation) are given for only those subjects for whom acceleration was above threshold.

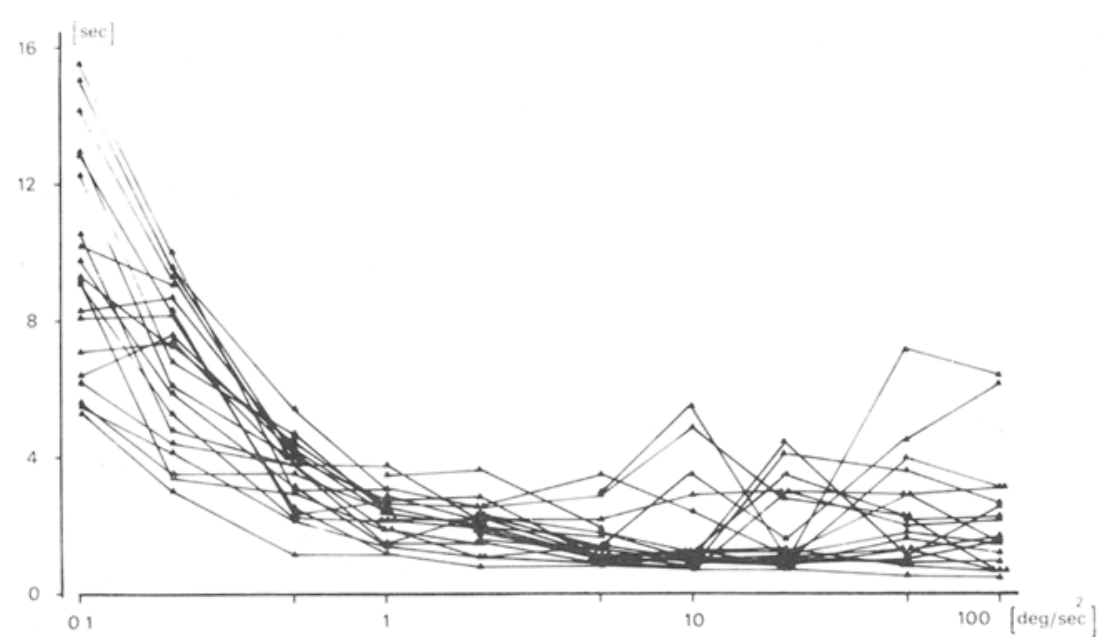

Figure 3. Latencies for the detection of motion during different degrees of accelerations for all subjects. Data points of individual subjects are connected by straight lines. There is a minimum in latency at $5 \mathrm{deg} / \mathrm{sec}^{2}$, with an average value of $1.4 \mathrm{sec}$. 

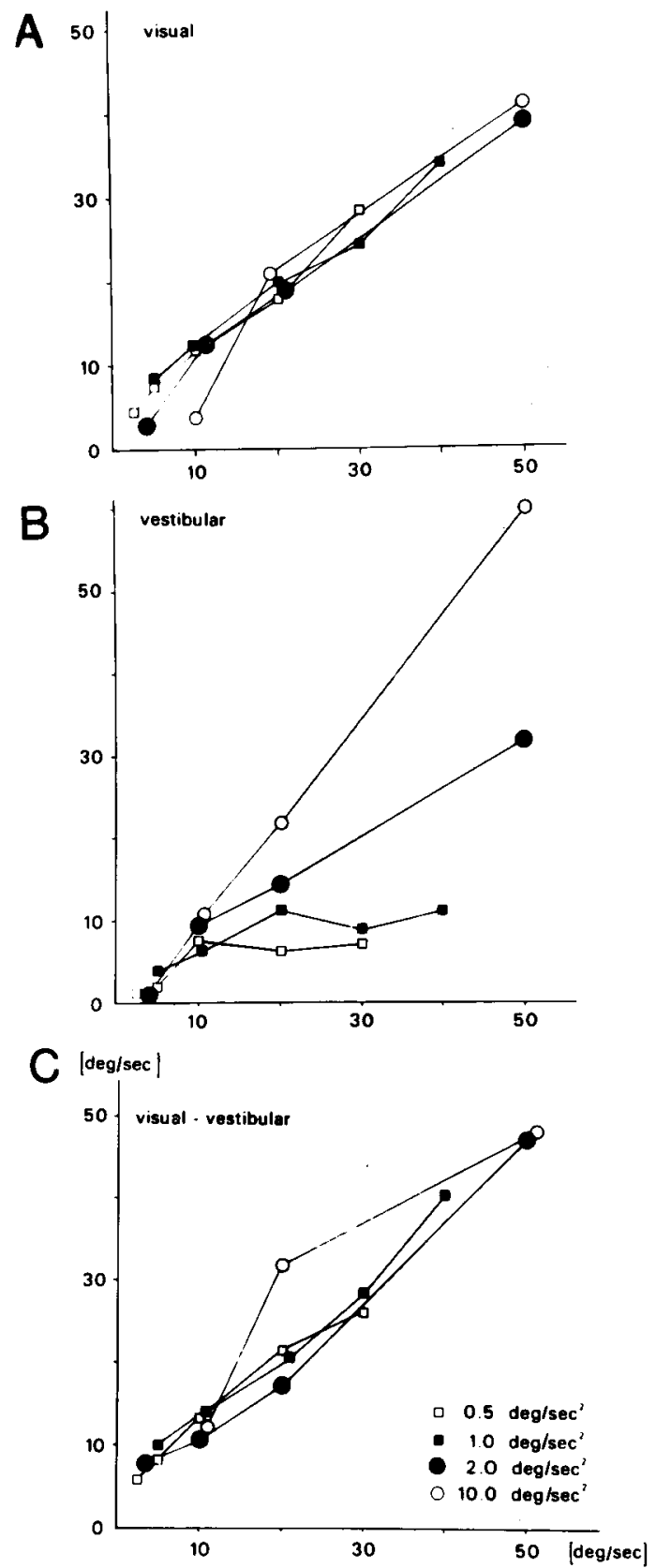

Figure 4. Subjective velocity sensation from six subjects during different accelerations with optokinetic (A), vestibular (B), and combined (C) stimulation. The abscissa gives instantaneous stimulus velocity, the ordinate, the subjective velocity. Average values obtained with different degrees of acceleration are connected by straight-line segments. Note that different accelerations were used, but that data points are plotted on a velocity scale.

well below unity. For all accelerations, subjective estimates remain below unity at velocities above $30 \mathrm{deg}$ / sec.

Vestibular stimulation. With vestibular stimulation and high accelerations, subjective motion corre- sponds well with actual velocity (Figure 4B). For low accelerations, however, subjective sensation is much below unity.

Combined stimulation. With all accelerations, subjective velocity estimation matches actual velocity more closely with combined stimulation than with optokinetic or vestibular stimulation alone (Figure 4C). Average values of up to about $60 \mathrm{deg} / \mathrm{sec}$ are near unity. At higher velocities, there is a consistent trend to underestimate velocities (not shown in figure).

\section{DISCUSSION}

The majority of our subjects ( 20 of 22) were experimentally naive; they were astonished and amused to experience CV. All subjects gave a consistent and similar indication of latency and amount of self-motion in response to the different forms of stimulation. At the lowest tested value $\left(.1 \mathrm{deg} / \mathrm{sec}^{2}\right.$ visual acceleration), latency for the detection of motion averaged about $9.7 \mathrm{sec}$. It decreased monotonically with increasing acceleration, up to about $5 \mathrm{deg} / \mathrm{sec}^{2}$. Conceptually, it is important to note that any motion with accelerations up to $5 \mathrm{deg} / \mathrm{sec}^{2}$ was always interpreted as full CV by all subjects. Only at accelerations above $5 \mathrm{deg} / \mathrm{sec}^{2}$ did subjects increasingly first experience object motion, which then gradually, over 1-2 sec, transformed into CV. An extreme visual acceleration would be the suddenly introduced exposure of the moving cylinder by switching lights on, enabling the subject to see a visual environment moving at constant velocity as was done by Brandt et al. (1973). Under these conditions, latencies for $\mathrm{CV}$ are about 3-4 sec.

In comparison, latencies to vestibular stimulation with the same acceleration values were consistently longer below $5 \mathrm{deg} / \mathrm{sec}^{2}$ or motion was not sensed at all, depending on individual thresholds. These acceleration values varied between less than .1 and $2 \mathrm{deg}$ / $\mathrm{sec}^{2}$ in our sample and are similar to those reported in the literature (Clark, 1967; Guedrey, 1974). Above $10 \mathrm{deg} / \mathrm{sec}^{2}$, latencies for vestibular stimulation are clearly shorter than those for CV. In measuring a subjective response, we included in the latency the reaction time to the stimulus, which can be estimated to be below $200 \mathrm{msec}$ and is therefore much smaller than the latencies measured.

These measurements support the notion that the subjective sensation of motion relies on different inputs, two main contributors being the visual and the peripheral vestibular system. The visual system is tuned to low accelerations and constant velocities, whereas the vestibular system is tuned to high accelerations (for a general review, see Henn et al., 1980). Since the amount of the contribution made by the different sensory channels depends on the values of acceleration and velocity, a nonlinear interaction has been proposed (Zaccharias \& Young, 1981). The re- 
sults of the combined stimulation would also support such an interpretation, since those results were consistently better than those obtained with either stimulation alone.

In the suprathreshold range, a similar pattern evolved: with low accelerations of the environment, magnitude of CV closely matched actual velocity. With high accelerations, part of the motion was interpreted as object motion and CV lagged actual velocity. With vestibular stimulation, an inverse pattern was observed. With high accelerations, there was a close relationship between actual and perceived velocity, whereas subjective responses were consistently too small at low accelerations. Only during combined stimulation did curves emerge with the subjective response matching actual stimulus velocity over the widest possible range and independent of the value or duration of the acceleration, although there was a systematic underestimation with increasing velocities. A similar interpretation has been given in experiments undertaken to explain the latency and magnitude of linear-vection, that is, sensation of motion during linear acceleration of the visual surround (Berthoz et al., 1975; also in Henn et al., 1980).

The results obtained from animal experiments are similar: Unit activity in the vestibular nuclei as well as nystagmus measurements under comparable conditions give virtually the same results (Waespe \& Henn, 1979; Waespe et al., 1980). In unit recordings from the alert monkey, even quantitative details are comparable: central vestibular responses to an optokinetic stimulus are proportional to stimulus accelerations up to about $5 \mathrm{deg} / \mathrm{sec}^{2}$ and lag actual velocity at higher accelerations. The nystagmus response is usually faster and shows no stimulus lag at the accelerations tested.

For humans, even with high-velocity exposure, buildup of nystagmus velocity can occur during virtually the first detectable slow phase for sudden exposures of stimulus velocities of more than $100 \mathrm{deg} /$ sec (Cohen et al., 1981). This again shows that nystagmus as a motor reflex response must be separated from the sensation of motion, although the two often go together.

Our results show that the subjective sensation of motion relies on visual input in an acceleration range up to about $5 \mathrm{deg} / \mathrm{sec}^{2}$ and on peripheral vestibular input in the range above $5 \mathrm{deg} / \mathrm{sec}^{2}$. The working ranges of both inputs overlap. Only if the inputs are combined does subjective sensation closely match actual velocity over the whole range of naturally occurring head or body movements. The experiments show how a simple paradigm can be tested by measuring subjective responses in humans, and nystagmus and single cell recordings in monkeys, all of which give comparable results.

\section{REFERENCES}

Berthoz, A., Pavard, B., \& Young, L. R. Perception of linear horizontal self-motion induced by peripheral vision (linearvection). Basic characteristics and visual-vestibular interactions. Experimental Brain Research, 1975, 23, 471 -489.

Brandt, Th., Dichanns, J., \& Koenig, E. Differential effects of central versus peripheral vision on egocentric and exocentric motion perception. Experimental Brain Research, 1973, 16, 476-491.

Clark, B. Thresholds for the perception of angular acceleration in man. Aerospace Medicine, 1967, 38, 443-450.

Cohen, B., Henn, V., Raphan, T., Dennett, D. Velocity storage, nystagmus, and visual-vestibular interactions in humans. Proceedings of the New York Academy of Sciences, 1982, in press.

Dichgans, J., \& BrandT, Th. Visual-vestibular interaction. Effects on self-motion perception and postural control. In R. Held, H. Leibowitz, H.-L. Teuber (Eds.), Handbook of sensory physiology (Vol. 8). Heidelberg: Springer, 1978.

Fischer, M. H., \& Kornmüller, A. E. Optokinetisch ausgelöste Bewegungswahrnehmungen und optokinetischer Nystagmus. Journal für Psychologie und Neurologie, 1930, 41, 273-308.

Guedrey, F. E. Psychophysics of vestibular sensation. In H. H. Kornhuber (Ed.), Handbook of sensory physiology (Vol. 6). Heidelberg: Springer, 1974.

Henn, V., Cohen, B., \& Young, L. R. Visual-vestibular interaction in motion perception and the generation of nystagmus. Neuroscience Research Program Bulletin, 1980, 18, 457-651.

MACH, E. Grundlinien der Lehre von den Bewegungsempfindungen. Amsterdam, Bonset, 1967. (Originally published, Leipzig, Engelmann, 1875.)

WAESPE, W., \& HENN, V. Motion information in the vestibular nuclei of alert monkeys: Visual and vestibular input vs optomotor output. In R. Granit \& P. Pompeiano (Eds.), Progress in brain research (Vol. 50): Reflex control of posture and movement. Amsterdam: Elsevier/North-Holland Biomedical Press, 1979.

Waespe, W., Henn, V., \& Isovitta, V. Nystagmus slow-phase velocity during vestibular, optokinetic, and combined stimulation in the monkey. Archiv für Psychiatrie und Nervenkrankheiten, $1980,228,275-286$.

Zacharias, G. L., \& Young, L. R. Influence of combined visual and vestibular cues on human perception and control of horizontal rotation. Experimental Brain Research, 1981, 41, 159-171.

(Manuscript received April 13, 1981; revision accepted for publication September 24, 1981.) 